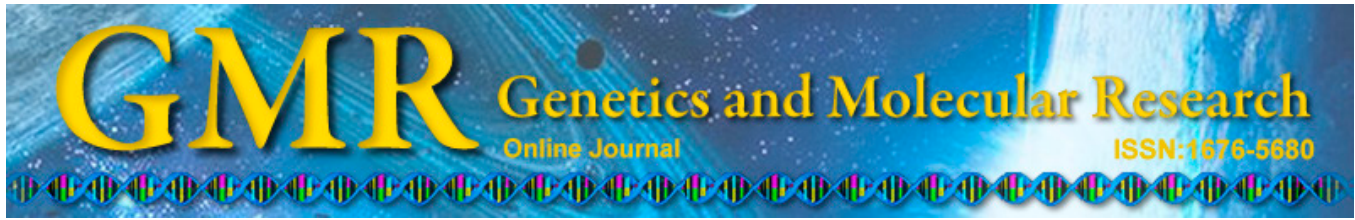

\title{
Correlation between promoter methylation in the GSTP1 gene and hepatocellular carcinoma development: a meta-analysis
}

\author{
Q.F. Li ${ }^{1}$, Q.Y. Li ${ }^{1}$, A.R. Gao ${ }^{2}$ and Q.F. Shi ${ }^{1}$ \\ ${ }^{1}$ Department of Infectious Diseases, Liao Cheng People's Hospital, \\ Liaocheng, China \\ ${ }^{2}$ Department of Obstetrics and Gynecology, Third Hospital of Liaocheng, \\ Liaocheng, China \\ Corresponding author: Q.F. Li \\ E-mail: liqingfang614@126.com
}

Genet. Mol. Res. 14 (2): 6762-6772 (2015)

Received August 28, 2014

Accepted February 6, 2015

Published June 18, 2015

DOI http://dx.doi.org/10.4238/2015.June.18.19

\begin{abstract}
Epigenetic silencing of the GSTP1 gene by promoter methylation has been associated with increased risk and shortened survival in patients with hepatocellular carcinoma (HCC). We therefore conducted a meta-analysis to obtain a more precise estimate of this association. By searching the Cochrane Library, CBM, EMBASE, PubMed, and the Web of Science, we tabulated and analyzed parameters from each study. Results were summarized by meta-analyses using the version 12.0 STATA software. Odds ratios (ORs) and 95\% confidence intervals $(95 \% \mathrm{CIs})$ were also calculated in this analysis. A total of 14 cohort studies (tumor samples $=607$, adjacent samples $=356$, benign samples $=182$, normal samples $=133$ ) were included for the following statistical analysis. Our meta-analysis results demonstrated that the frequency of GSTP1 methylation in cancer tissues was significantly higher than those in adjacent tissues, benign tissues, and normal tissues (all $\mathrm{P}<0.05)$. Further subgroup analysis by country indicated that the frequency of aberrant GSTP1 promoter methylation was correlated
\end{abstract}


to the development of HCC among all the included experimental subgroups (all $\mathrm{P}<0.05$ ). The results indicate a significant association between GSTP1 methylation and poor outcomes in HCC patients.

Key words: GSTP1; Promoter methylation; Hepatocellular carcinoma; Meta-analysis

\section{INTRODUCTION}

Hepatocellular carcinoma (HCC) is the fifth most frequent malignancy in men $(523,000$ cases per year, $7.9 \%$ of all cancers) and women (226,000 cases per year, $6.5 \%$ of all cancers) globally (El-Serag, 2012). According to statistics from the American Cancer Society, HCC also produces a high mortality rate in both men and women; it was one of the top ten causes of cancerous death in the United States in 2013 (Siegel et al., 2013). Furthermore, most of the burden of HCC is borne by developing countries, and it is estimated that about half of HCC cases and deaths occur in China (Jemal et al., 2011). As a multifunctional disease, HCC is thought to be caused by the interaction of genetic and environmental factors (Clifford et al., 2010; Trichopoulos et al., 2011). The majority of cases of HCC are correlated with chronic hepatitis $\mathrm{B}$ virus or hepatitis $\mathrm{C}$ virus infections, while heavy alcohol consumption, tobacco smoking, obesity, cirrhosis, aflatoxins, and fatty liver disease may also contribute to increased risk of HCC (Schütte et al., 2009; El-Serag, 2012). In addition to those environmental risk factors, there is some evidence that genetic factors are also potentially correlated with the progression of HCC (Long et al., 2009; Weng et al., 2010). Recently, several studies have suggested that DNA methylation in the detoxifying genes, including the glutathione S-transferase pi 1 gene (GSTP1), may be closely related to increased risk of HCC (Lambert et al., 2011; Jain et al., 2012).

Human GSTs are a superfamily of phase II drug-metabolizing enzymes. They feature significantly in the protection of cells against xenobiotics and oxidative stress by catalyzing the conjugation of electrophilic compounds to glutathione in the detoxification process (Oakley, 2011; Dusinska et al., 2012). In view of their biochemical, immunologic, and structural properties, the GST family is categorized into four key classes: A (alpha), M (mu), P (pi), and T (theta) (Magno et al., 2009). GSTP is a member of the GST family and is encoded by the human GSTP1 gene. The locus of the gene is 11q13, it is 3-kilobases (kb) long, and comprises 6 introns and 7 exons (Strange et al., 2001). The GSTP1 protein plays a critical role in the process of detoxification, which can protect normal cells against damage by various carcinogens or electrophilic compounds (Masood and Kayani, 2013). Abnormal activity and overexpression of GSTP1 have been reported in many neoplasms, while GSTP1 genetic polymorphism and DNA hyper-methylation have also been observed in several cancers, including breast, lung, and liver cancers (Sakoda et al., 2008; Carlsten et al., 2008; Chen et al., 2010). In addition, epigenetic inactivation of GSTP1 may result in the decline or even loss of the detoxification function of GSTP1, and carcinogens can lead to hepatocyte damage; thus, GSTP1 may be closely related to cancer initiation (Lambert et al., 2011). In addition, abnormal CpG island methylation is one of the main molecular mechanisms of GSTP1 inactivation in some types of cancer. In the past few decades, studies have shown that promoter hyper-methylation of GSTP1 often occurs in human hepatitis B virus-related diseases, and can be detected in early-stage HCC tumor tissues (Tischoff and Tannapfe, 2008; Rongrui et al., 2014). In this regard, GSTP1 promoter methyla- 
tion may be involved in the occurrence and development of HCC (Wang et al., 2006; Kiran et al., 2009). However, there are also contradictory results concerning the role of GSTP1 promoter methylation in the progression of HCC (Hua et al., 2011; Jain et al., 2012). Given the conflicting evidence on this issue, we performed a meta-analysis using all available data to evaluate the exact relationship between GSTP1 promoter methylation and increased risk of HCC in order to find a potential biomarker for early clinical diagnosis of HCC.

\section{MATERIAL AND METHODS}

\section{Literature search and selection criteria}

We systemically searched for studies, without language restrictions, in numerous electronic databases: the Cochrane Library (Issue 12, 2013), CBM (1982-2013), EMBASE (19802013), PubMed (1966-2013), and Web of Science (1945-2013). The searching strategy was performed using "Methyltransferases", "methylation" or "DNA Methylation", "Glutathione S-Transferase pi" and "Carcinoma, Hepatocellular" as the medical subject headings (MeSH), and was accompanied by the following free text word searching terms: ("Hypermethylation" or "Demethylation" or "methylation"); ("Glutathione S-Transferase pi" or "GST Class-phi" or "Glutathione Transferase P1-1" or "GSTP1 Glutathione D-Transferase" or "GSTP1 Glutathione D Transferase" or "GSTP1"); and ("hepatocellular cancer" or "hepatocellular neoplasms" or "liver carcinoma" or "liver cancer" or "liver neoplasms" or "HCC" or "liver tumor" or "hepatoma carcinoma" or "hepatoma cancer" or "hepato carcinoma" or "Liver Cell Carcinoma" or "hepatocellular carcinoma"). Titles and abstracts among the initially identified articles were evaluated for appropriateness.

Inclusion criteria used to identify the eligibility of included studies were as follows: 1) the patients were limited to HCC without restriction of types; 2) methods suitable for the methylation detection of the GSTP1 gene were confined to methylation-specific polymerase chain reaction (MSP), methylation-sensitive restriction enzymes-quantitative polymerase chain reaction (MSRE-qPCR), and MethyLight array; 3) original articles with cohort design; 4) studies had to be about the correlation between GSTP1 promoter methylation and the risk and prognosis of HCC; 5) all patients diagnosed with HCC must have been confirmed by histopathologic examinations; 6) sufficient information regarding the comparison of HCC tissues with adjacent, benign, and normal tissues must have been provided in the article. In addition, if reported in duplicate, the most recent article published in English or the study with the largest sample size was included in this study.

\section{Data extraction and methodological assessment}

Each included study was reviewed by two observers using a standardized form to systematically collect relevant data. For all studies, we extracted and recorded the following data from original publications: surname and title, first author, year of publication, source of publication, study type, study design, age, gender, ethnicity, and country of origin. Design of study, total number of cases, source of subjects, type of sample, sample size, detection method of methylation, and frequency of methylation were also included.

To ensure consistency in reviewing and reporting results, two reviewers independent- 
ly assessed the methodological quality of the included trials using the Newcastle-Ottawa scale criteria (Stang, 2010). The three broad perspectives judged were: 1 ) subject selection $(0-4) ; 2)$ subject comparability (0-2); and 3 ) clinical outcome (0-3). The Newcastle-Ottawa scale scores ranged from 0 to 9 ; a study was deemed good quality if the score was $\geq 7$.

\section{Statistical analysis}

The odds of GSTP1 methylation in HCC tissue versus adjacent, bening, and normal tissues are reported as odds ratios (ORs) with their corresponding $95 \%$ confidence intervals (95\% CIs) using the Z-test. Between-study heterogeneity was assessed by the Cochran Q-statistic and $\mathrm{I}^{2}$ tests (Zintzaras and Ioannidis, 2005). Heterogeneity across the enrolled studies was evaluated by the Cochran Q-statistic test (Q-test exhibited a P $<0.05$ or the $\mathrm{I}^{2}$ test showed $>50 \%$ ). Ultimately, the random-effect model was applied when heterogeneity existed among studies, while the fixed-effect model was applied when there was no statistical heterogeneity. The subgroup meta-analyses were also conducted by country and method to explore potential effect modification. One-way sensitivity analysis was performed to investigate whether results were affected significantly by removing single studies one at a time. Potential publication bias was investigated using funnel plots and the Egger linear regression test (Peters et al., 2006). We used the version 12.0 STATA statistical software to achieve a rigorous statistical metaanalysis.

\section{RESULTS}

\section{Baseline characteristics of studies included}

The original search yielded a total of 103 papers related to the searched keywords.

According to the inclusion criteria, 14 cohort studies were included (Zhong et al., 2002; Lee et al., 2003; Tada et al., 2005; Wang et al., 2006; Zhang et al., 2006; Su et al., 2007; Harder et al., 2008; Chang et al., 2008; Moribe et al., 2009; Kiran et al., 2009; Li et al., 2010; Feng et al., 2010; Hua et al., 2011; Jain et al., 2012) and 89 articles were excluded. A total of 607 HCC samples in 14 cohort studies were included; additionally, 356 adjacent samples, 182 benign samples, and 133 normal samples were included. The publication year of the studies ranged from 2002 to 2012.

The geographic setting of the studies was heterogeneous: two studies were from the USA, seven were from China, three were from Japan, and there was one study each from Germany and Korea. The detected samples used for examination of the promoter methylation of GSTP1 were extracted from cancer tissues in the included studies. The MSP method used for this meta-analysis was adopted in ten studies, and the other four studies were conducted using non-MSP methods, including MSRE-qPCR, MethyLight array, and quantitative methylation-specific polymerase chain reaction (qMSP) methods. Table 1 summarizes the characteristics and methodological quality of the studies enrolled.

\section{Quantitative data synthesis}

Fourteen studies were included to assess the potential role of GSTP1 promoter methylation in the development of HCC in patients. A random-effect model was used where heterogeneity existed $(\mathrm{P}<0.05)$. Our findings demonstrate that the frequency of the GSTP1 gene 
methylation in cancer tissues is significantly higher than in adjacent, benign, and normal tissues (cancer tissue $v s$ adjacent tissue: $\mathrm{OR}=8.49,95 \% \mathrm{CI}=6.15-11.71, \mathrm{P}<0.001$; cancer tissue $v s$ benign tissue: $\mathrm{OR}=6.54,95 \% \mathrm{CI}=4.73-9.04, \mathrm{P}<0.001$; cancer tissue $v s$ normal tissue: $\mathrm{OR}$ $=10.64,95 \% \mathrm{CI}=7.67-14.74, \mathrm{P}<0.001)($ Figure 1$)$.

Table 1. Characteristics of included studies focused on methylation ratio of GSTP1.

\begin{tabular}{|c|c|c|c|c|c|c|c|c|c|c|c|}
\hline \multirow[t]{2}{*}{ First author } & \multirow[t]{2}{*}{ Year } & \multirow[t]{2}{*}{ Country } & \multirow[t]{2}{*}{ Sample } & \multicolumn{4}{|c|}{ Number } & \multirow{2}{*}{$\begin{array}{c}\text { Gender } \\
(\mathrm{M} / \mathrm{F})\end{array}$} & \multirow{2}{*}{$\begin{array}{c}\text { Age } \\
\text { (years) }\end{array}$} & \multirow[t]{2}{*}{ Method } & \multirow{2}{*}{$\begin{array}{l}\text { NOS } \\
\text { score }\end{array}$} \\
\hline & & & & Tumor & Adjacent & Benign & Normal & & & & \\
\hline Jain S & 2012 & USA & 56 & 20 & 20 & 10 & 6 & $11 / 9$ & $60.2 \pm 12.4$ & MSP & 7 \\
\hline Hua D & 2011 & China & 102 & 47 & 47 & 0 & 8 & $36 / 11$ & $55(27-78)$ & MSRE-qPCR & 8 \\
\hline $\mathrm{Li} \mathrm{B}$ & 2010 & China & 221 & 115 & 48 & 48 & 10 & $84 / 31$ & $50(27-76)$ & MSP & 8 \\
\hline Feng Q & 2010 & USA & 65 & 40 & 0 & 0 & 25 & $32 / 8$ & $53.7 \pm 9.3$ & MethyLight array & 6 \\
\hline Moribe $\mathrm{T}$ & 2009 & Japan & 48 & 25 & 20 & 0 & 3 & $32 / 13$ & - & qMSP & 6 \\
\hline Kiran M & 2009 & Japan & 43 & 23 & 0 & 20 & 0 & - & 59 & MSP & 6 \\
\hline Harder J & 2008 & Germany & 84 & 34 & 0 & 34 & 16 & $27 / 8$ & $59(40-72)$ & qMSP & 7 \\
\hline Chang $\mathrm{H}$ & 2008 & China & 36 & 19 & 0 & 17 & 0 & - & - & MSP & 6 \\
\hline Su PF & 2007 & China & 172 & 58 & 58 & 11 & 45 & $54 / 4$ & $56(29-85)$ & MSP & 8 \\
\hline Zhang YC & 2006 & China & 106 & 53 & 53 & 0 & 0 & $43 / 10$ & $57(29-66)$ & MSP & 8 \\
\hline Wang J & 2006 & China & 60 & 26 & 26 & 8 & 0 & - & - & MSP & 7 \\
\hline Tada M & 2005 & Japan & 88 & 44 & 44 & 0 & 0 & $40 / 4$ & $62.3 \pm 11.0$ & MSP & 6 \\
\hline Lee $\mathrm{S}$ & 2003 & Korea & 114 & 60 & 0 & 34 & 20 & $47 / 13$ & 54 & MSP & 8 \\
\hline Zhong S & 2002 & China & 83 & 43 & 40 & 0 & 0 & - & - & MSP & 7 \\
\hline
\end{tabular}

$\mathrm{M}=$ male; $\mathrm{F}=$ female; NOS $=$ Newcastle-Ottawa Scale.

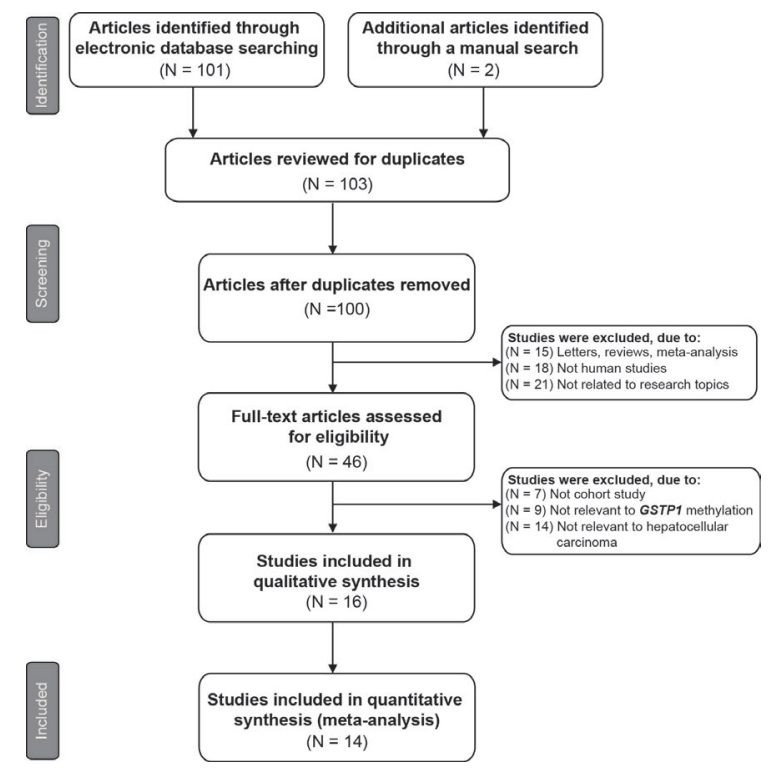

Figure 1. Flow chart shows study selection procedure. Fourteen cohort studies were included in this meta-analysis.

We also performed subgroup analyses in order to further explore the association between GSTP1 methylation and HCC risk across different subgroups. Further subgroup analysis based on country showed that the aberrant methylation of GSTP1 in cancer tissue was significantly related to the development of HCC in patients compared with adjacent, benign, and normal tissues among USA, Chinese, Japanese, German, and Korean populations (all P values <0.05) (Figure 2). 


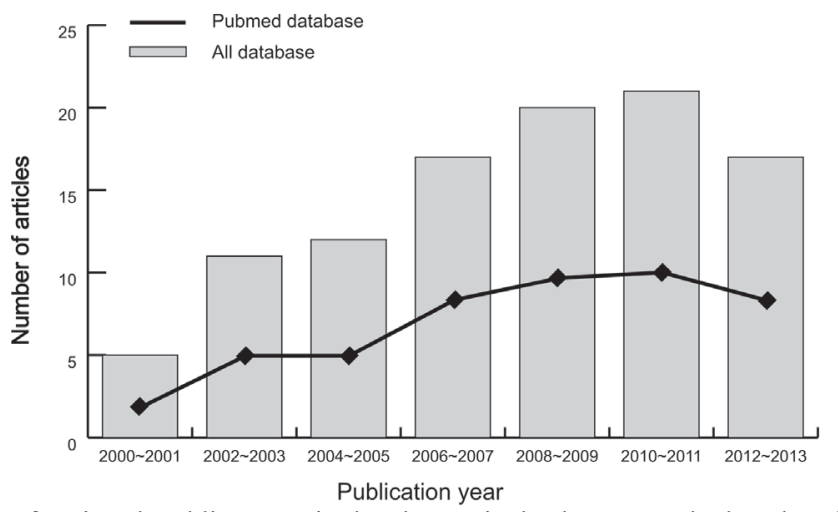

Figure 2. Distribution of topic-related literature in the electronic database over the last decade.

Furthermore, in the subgroup analysis based on method, we observed an association between the aberrant methylation of GSTP1 in the cancer tissues and HCC progression compared with adjacent, benign, and normal tissues among both the MSP and non-MSP method subgroup members (all $\mathrm{P}$ values $<0.05$ ) (Figure 2). A sensitivity analysis was performed and the analysis results indicated that no individual study significantly affected the overall pooled estimates (Figure 3).
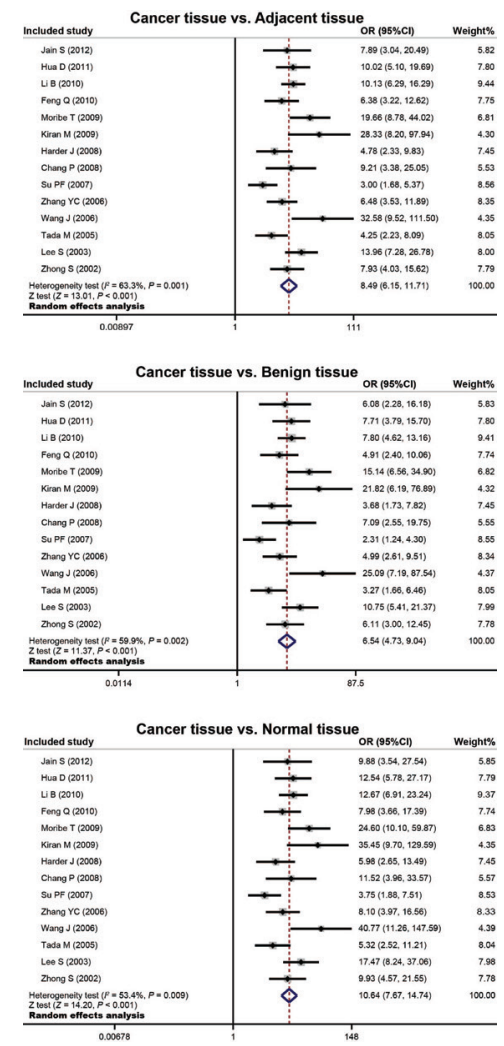

Figure 3. Forest plots for the relationship between GSTP1 promoter methylation and hepatocellular carcinoma. 
The graphical funnel plots of the 14 studies presented no apparent asymmetry, and the Egger test showed no publication bias in our current meta-analysis (all P values $>0.05$ ) (Figure 4).
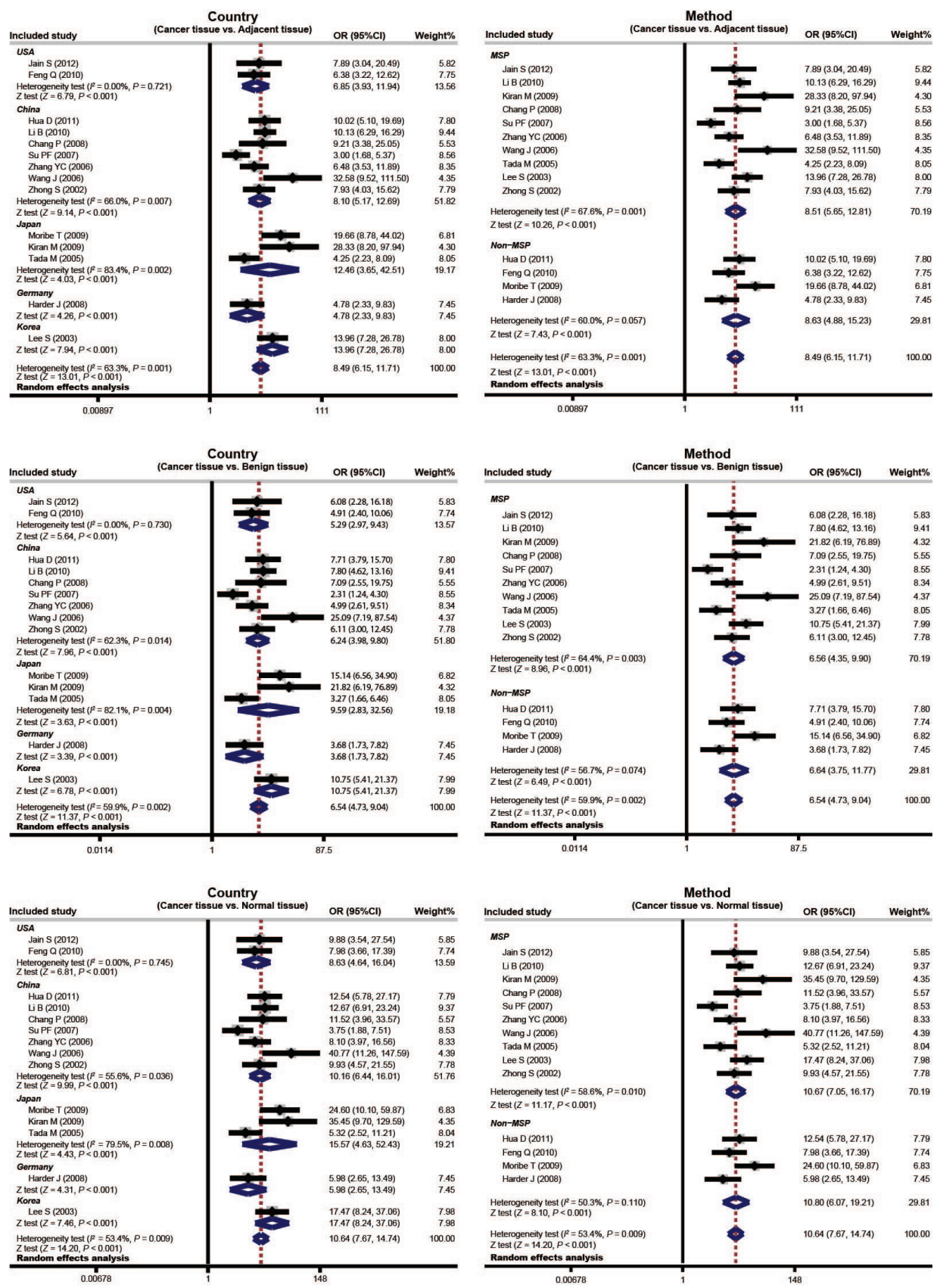

Figure 4. Subgroup analyses for the relationships between GSTP1 promoter methylation and hepatocellular carcinoma. 


\section{DISCUSSION}

In this meta-analysis, by systematically reviewing all the relevant prospective cohort studies we attempted to provide a better understanding of the relationship between the aberrant methylation of GSTP1 and the development and progression of HCC. The cumulative analyses from the 14 cohorts showed that the aberrant methylation of GSTP1 was observed in cancer tissue but rarely in adjacent, benign, and normal tissue, suggesting that GSTP1 hypermethylation can be considered a possible candidate for predicting the development of HCC in patients. It must be recognized that epigenetic silencing of tumor suppressor genes by promoter methylation plays an important role in regulation of gene expression, control of development, and other life activities (Ben Ayed-Guerfali et al., 2011). GSTP1 acts as an important tumor suppressor gene, and it is generally accepted that investigation of the hyper-methylation of GSTP 1 may elucidate the mechanisms of carcinogenesis (Richiardi et al., 2009). Although the aberrant methylation of GSTP 1 has also been described in a substantial proportion of hepatocarcinogenesis cases (Feng et al., 2010; Lambert et al., 2011), details about the mechanisms and significance of DNA hyper-methylation of GSTP1 in HCC are still unknown. The mature GSTP1 protein functions as a detoxifying enzyme, which may play a role in carcinogenesis (Chang et al., 2008). However, variations in the degree of DNA methylation may influence the expression of multiple cancer-related genes, thereby altering cancer progression; hypermethylation of the GSTP1 gene may, therefore, have an effect that is equivalent to genetic mutations that activate the gene (Kiran et al., 2009; Feng et al., 2010). In general, differential CpG island methylation of the GSTP1 gene seems to be the initial event in hepatocarcinogenesis; it is responsible for the loss of expression and function of GSTP1, and is related to transcriptional inactivation (Chang et al., 2008). Therefore, our findings suggest that GSTP1 promoter methylation is a critical event in relation to the progression of HCC. In addition, recent advances have shown that aberrant methylation of GSTP1 may also be regarded as an emerging biomarker for diagnosis and surveillance of people at high risk of HCC (Hua et al., 2011). Moreover, the GSTP1 promoter may also contribute to distinguishing HCC from cirrhosis and hepatitis, where the MSP assay would have poor specificity. The existing evidence may not only validate our hypothesis that the methylation of GSTP1 affects its specificity as a marker for HCC screening, but may also provide an explanation for the differences that distinguish HCC tissue from other liver-disease tissues (Jain et al., 2012). In accordance with our results, one previous study showed that $\mathrm{HCC}$ features a high frequency of $\mathrm{CpG}$ island hyper-methylation in the promoter regions; the aberrant hyper-methylation of GSTP1 tends to accelerate during the multistep process of hepatocarcinogenesis, and might be a possible means of predicting the development of HCC, especially in the premalignant stages (Lee et al., 2003). Similarly, Li et al. (2010) concluded that CpG island methylation of GSTP1 may specifically define a subgroup of patients with unfavorable outcomes in TNM stage I HCC, suggesting that the examination of GSTP1 methylation may be useful for stratifying prognosis of patients with early-stage HCC and identifying patients who are at higher risk of recurrence.

Considering that myriad factors may potentially influence the prognosis of gastric cancer patients, we further performed a stratified analysis based on country. Subgroup analysis in this fashion indicated that aberrant methylation of GSTP1 in cancer tissue was significantly related to development of $\mathrm{HCC}$ compared with adjacent, benign, and normal tissues among USA, Chinese, Japanese, German, and Korean populations. One possible interpretation may be that hyper-methylation of GSTP1 is related to loss of gene function and is intimately in- 
volved in the early process of HCC carcinogenesis and tumor progression (Moribe et al., 2009). In conclusion, our results are partially consistent with the previous hypothesis that the aberrant methylation of GSTP 1 may be conducive to the progression of $\mathrm{HCC}$, suggesting that GSTP1 gene methylation may represent a marker for identifying individuals who are at high risk of developing HCC.

Simultaneously, a number of potential limitations have also been taken into account in our meta-analysis. First, and most importantly, is the existence of heterogeneity. In the current meta-analysis, only a small portion of the heterogeneity could be attributed to the differences between different countries and ethnic backgrounds, or differences in the methods used. This suggests that other sources of heterogeneity must have been present among the included studies. A second limit of this analysis may have been publication and reporting bias. We attempted to use several Chinese and English databases that were as complete as possible to minimize publication bias. However, we did not take unpublished papers and abstracts into account entirely. Third, in some of the included literature, the number of HCC patients was relatively small, which may have restricted the outcome of this meta-analysis. In addition, it is difficult to establish the cut-off point of hyper-methylation for predicting cancer risk, and therefore the sensitivity and specificity of gene methylation with respect to cancer risk was not easy to determine.

In conclusion, the findings of our meta-analysis highlight the potential value of GSTP1 methylation for predicting the pathogenesis and prognosis of HCC in patients. Thus, detection of GSTP1 methylation may be a beneficial and valuable biomarker for early diagnosis of HCC. However, heterogeneity may be an important factor that has an adverse effect on the conclusion of this outcome. It is therefore essential to produce a more extensive large-scale study in the future.

\section{Conflicts of interest}

The authors declare no conflict of interest.

\section{ACKNOWLEDGMENTS}

We would like to acknowledge the reviewers for their helpful comments on this paper.

\section{REFERENCES}

Ben Ayed-Guerfali D, Benhaj K, Khabir A, Abid M, et al. (2011). Hypermethylation of tumor-related genes in Tunisian patients with gastric carcinoma: clinical and biological significance. J. Surg. Oncol. 103: 687-694.

Carlsten C, Sagoo GS, Frodsham AJ, Burke W, et al. (2008). Glutathione S-transferase M1 (GSTM1) polymorphisms and lung cancer: a literature-based systematic HuGE review and meta-analysis. Am. J. Epidemiol. 167: 759-774.

Chang H, Yi B, Li L, Zhang HY, et al. (2008). Methylation of tumor associated genes in tissue and plasma samples from liver disease patients. Exp. Mol. Pathol. 85: 96-100.

Chen YL, Tseng HS, Kuo WH, Yang SF, et al. (2010). Glutathione S-Transferase P1 (GSTP1) gene polymorphism increases age-related susceptibility to hepatocellular carcinoma. BMC Med. Genet. 11: 46.

Clifford RJ, Zhang J, Meerzaman DM, Lyu MS, et al. (2010). Genetic variations at loci involved in the immune response are risk factors for hepatocellular carcinoma. Hepatology 52: 2034-2043.

Dusinska M, Staruchova M, Horska A, Smolkova B, et al. (2012). Are glutathione S transferases involved in DNA damage signalling? Interactions with DNA damage and repair revealed from molecular epidemiology studies. Mutat. Res. 736: $130-137$. 
El-Serag HB (2012). Epidemiology of viral hepatitis and hepatocellular carcinoma. Gastroenterology 142: 1264-1273.

Feng Q, Stern JE, Hawes SE, Lu H, et al. (2010). DNA methylation changes in normal liver tissues and hepatocellular carcinoma with different viral infection. Exp. Mol. Pathol. 88: 287-292.

Harder J, Opitz OG, Brabender J, Olschewski M, et al. (2008). Quantitative promoter methylation analysis of hepatocellular carcinoma, cirrhotic and normal liver. Int. J. Cancer 122: 2800-2804.

Hua D, Hu Y, Wu YY, Cheng ZH, et al. (2011). Quantitative methylation analysis of multiple genes using methylationsensitive restriction enzyme-based quantitative PCR for the detection of hepatocellular carcinoma. Exp. Mol. Pathol. 91: 455-460.

Jain S, Chen S, Chang KC, Lin YJ, et al. (2012). Impact of the location of CpG methylation within the GSTP1 gene on its specificity as a DNA marker for hepatocellular carcinoma. PLoS One 7: e35789.

Jemal A, Bray F, Center MM, Ferlay J, et al. (2011). Global cancer statistics. CA Cancer J. Clin. 61: 69-90.

Kiran M, Chawla YK and Kaur J (2009). Methylation profiling of tumor suppressor genes and oncogenes in hepatitis virus-related hepatocellular carcinoma in northern India. Cancer Genet. Cytogenet. 195: 112-119.

Lambert MP, Paliwal A, Vaissière T, Chemin I, et al. (2011). Aberrant DNA methylation distinguishes hepatocellular carcinoma associated with HBV and HCV infection and alcohol intake. J. Hepatol. 54: 705-715.

Lee S, Lee HJ, Kim JH, Lee HS, et al. (2003). Aberrant CpG island hypermethylation along multistep hepatocarcinogenesis. Am. J. Pathol. 163: 1371-1378.

Li B, Liu W, Wang L, Li M, et al. (2010). CpG island methylator phenotype associated with tumor recurrence in tumornode-metastasis stage I hepatocellular carcinoma. Ann. Surg. Oncol. 17: 1917-1926.

Long XD, Ma Y, Zhou YF, Yao JG, et al. (2009). XPD codon 312 and 751 polymorphisms, and AFB1 exposure, and hepatocellular carcinoma risk. BMC Cancer 9: 400.

Magno LA, Talbot J, Talbot T, Borges Santos AM, et al. (2009). Glutathione s-transferase variants in a brazilian population. Pharmacology 83: 231-236.

Masood N and Kayani MA (2013). Expression patterns of carcinogen detoxifying genes (CYP1A1, GSTP1 \& GSTT1) in HNC patients. Pathol. Oncol. Res. 19: 89-94.

Moribe T, Iizuka N, Miura T, Kimura N, et al. (2009). Methylation of multiple genes as molecular markers for diagnosis of a small, well-differentiated hepatocellular carcinoma. Int. J. Cancer 125: 388-397.

Oakley A (2011). Glutathione transferases: a structural perspective. Drug. Metab. Rev. 43: 138-151.

Peters JL, Sutton AJ, Jones DR, Abrams KR, et al. (2006). Comparison of two methods to detect publication bias in metaanalysis. JAMA 295: 676-680.

Richiardi L, Fiano V, Vizzini L, De Marco L, et al. (2009). Promoter methylation in APC, RUNX3, and GSTP1 and mortality in prostate cancer patients. J. Clin. Oncol. 27: 3161-3168.

Rongrui L, Na H, Zongfang L, Fanpu J, et al. (2014). Epigenetic mechanism involved in the HBV/HCV-related hepatocellular carcinoma tumorigenesis. Curr. Pharm. Des. 20: 1715-1725.

Sakoda LC, Blackston CR, Xue K, Doherty JA, et al. (2008). Glutathione S-transferase M1 and P1 polymorphisms and risk of breast cancer and fibrocystic breast conditions in Chinese women. Breast Cancer Res. Treat. 109: 143-155.

Schütte K, Bornschein J, Malfertheiner P (2009). Hepatocellular carcinoma - epidemiological trends and risk factors. Dig. Dis. 27: 80-92.

Siegel R, Naishadham D and Jemal A (2013). Cancer statistics, 2013. CA Cancer J. Clin. 63: 11-30.

Stang A (2010). Critical evaluation of the Newcastle-Ottawa scale for the assessment of the quality of nonrandomized studies in meta-analyses. Eur. J. Epidemiol. 25: 603-605.

Strange RC, Spiteri MA and Ramachandran S (2001). Glutathione-S-transferase family of enzymes. Mutat. Res. 482: 21-26.

Su PF, Lee TC, Lin PJ, Lee PH, et al. (2007). Differential DNA methylation associated with hepatitis B virus infection in hepatocellular carcinoma. Int. J. Cancer 121: 1257-1264.

Tada M, Yokosuka O, Fukai K, Chiba T, et al. (2005). Hypermethylation of NAD(P)H: quinone oxidoreductase 1 (NQO1) gene in human hepatocellular carcinoma. J. Hepatol. 42: 511-519.

Tischoff I and Tannapfe A (2008). DNA methylation in hepatocellular carcinoma. World J. Gastroenterol. 14: 1741-1748.

Trichopoulos D, Bamia C, Lagiou P, Fedirko V, et al. (2011). Hepatocellular carcinoma risk factors and disease burden in a European cohort: a nested case-control study. J. Natl. Cancer Inst. 103: 1686-1695.

Wang J, Qin Y, Li B, Sun Z, et al. (2006). Detection of aberrant promoter methylation of GSTP1 in the tumor and serum of Chinese human primary hepatocellular carcinoma patients. Clin. Biochem. 39: 344-348.

Weng CJ, Hsieh YH, Tsai CM, Chu YH, et al. (2010). Relationship of insulin-like growth factors system gene polymorphisms with the susceptibility and pathological development of hepatocellular carcinoma. Ann. Surg. Oncol. 17: 1808-1815.

Zhang YC, Chen YP, Chen JX and Deng CS (2006). Methylation of glutathione S-transferases P1 in hepatocellular carcinoma. Chin. J. Gastroenterol. Hepatol. 15: 399-404.

Genetics and Molecular Research 14 (2): 6762-6772 (2015) 
Zhong S, Tang MW, Yeo W, Liu C, et al. (2002). Silencing of GSTP1 gene by CpG island DNA hypermethylation in HBV-associated hepatocellular carcinomas. Clin. Cancer Res. 8: 1087-1092.

Zintzaras E and Ioannidis JP (2005). HEGESMA: genome search meta-analysis and heterogeneity testing. Bioinformatics 21: $3672-3673$. 